\title{
Endothelin-1 indicates unfavorable prognosis in primary high-grade non-muscle-invasive urothelial bladder cancer
}

\author{
Lampros Mitrakas $^{1}$, Stavros Gravas ${ }^{1}$, Foteini Karasavvidou ${ }^{2}$, Ioannis Zachos ${ }^{1}$, Anastasios Karatzas ${ }^{1}$, \\ Athanasios Oeconomou ${ }^{1}$, Georgios Koukoulis ${ }^{2}$, Vasilios Tzortzis ${ }^{1}$, Christos Papandreou ${ }^{3}$ \\ ${ }^{1}$ Department of Urology, Faculty of Medicine-School of Health Sciences-University of Thessaly, University Hospital \\ of Larissa, Greece; \\ ${ }^{2}$ Department of Pathology and Cytology, Faculty of Medicine-School of Health Sciences-University of Thessaly, \\ University Hospital of Larissa, Greece; \\ ${ }^{3}$ Department of Oncology, School of Medicine, Aristotle University of Thessaloniki, "N.Papageorgiou" Hospital of Thessaloniki, \\ Ring Road of Thessaloniki, Greece.
}

\begin{abstract}
Summary Objective: To conduct a prospective study of the potential prognostic role of endothelin-1 (ET-1) in a cohort of primary high-grade non-muscle-invasive urothelial bladder cancer patients, who were treated with adjuvant intravesical Bacillus Calmette-Guérin (BCG).

Material and methods: Patients with primary high-grade nonmuscle-invasive urothelial bladder cancer, who received postoperatively induction and maintenance BCG therapy, were prospectively included. Recurrence and progression were histologically proven. Immunohistochemical staining for ET-1 was assessed. Epidemiological, pathological and clinical parameters as well as the expression of ET-1 in tumor specimens were statistically analyzed for recurrence, progression, recurrence-free survival (RFS) and progression-free survival (PFS).

Results: ET-1 associates significantly with recurrence $(p=0.000)$, progression $(p=0.000), \operatorname{RFS}(p=0.000)$ and PFS $(p=0.000)$.

The patient's age is also significant for recurrence ( $p=0.003$, $O R=1.27395 \%$ CI: $1.086-1.492)$ and RFS $(p=0.013)$.

Conclusions: ET-1 seems to deteriorate prognosis in patients suffering from primary high-grade non-muscle-invasive urothelial bladder cancer, who are treated with adjuvant BCG instillations. Furthermore, the patient's age associates with an increased likelihood for recurrence.
\end{abstract}

KEY WORDS: ET-1; High-grade; Non-muscle-invasive bladder cancer; Prognosis.

Submitted 17 November 2020; Accepted 25 January 2021

\section{INTRODUCTION}

In urothelial bladder cancer about $75 \%$ of new patients are diagnosed with a non-muscle-invasive tumor.

The most effective adjuvant treatment for high-grade nonmuscle-invasive urothelial bladder cancer (HGNMIUBC) is the use of intravesical instillations of Bacillus CalmetteGuérin (BCG) (1). Unfortunately, recurrence and progression are documented in about $30 \%$ and $12 \%(0-35 \%)$ of these patients (2). Furthermore, pTlG3 patients have a 5 -year disease progression rate of $19.8 \%$ and a 5 -year disease-specific death rate of $11.3 \%$, which proves a poor prognosis (3). Radical cystectomy is the chosen treatment in cases of failure of BCG therapy. Therefore, the early iden- tification of the patients who will not respond to BCG, in order to implement an aggressive therapy, aiming to maximize the clinical benefit, is clinically important (4).

Endothelin-1 (ET-1) is a multifunctional peptide. ET-1 and its receptors A/ETAR and B/ETBR consist the endothelin-axis (ET axis), which plays a role in cancer biology. ET-1 stimulates tumor cell proliferation, facilitates tumor invasion and metastasis and has antiapoptotic and neoangiogenic effects (5). ETAR associates with tumor-cell proliferation and tumor progression, inhibition of apoptosis, effects on bone matrix, production of vascular endothelial growth factor (VEGF) leading to endothelial cell proliferation and vascular permeability, by increasing the levels of hypoxia-inducible factorla/HIF-la (5). ETBR inducts proliferation of endothelial cells and migration (5).

Our purpose is to conduct a prospective study of the potential prognostic significance of ET-1 in a cohort of primary HGNMIUBC patients who were treated postoperatively with BCG (induction as well as maintenance).

\section{MATERIAL AND METHOdS}

We prospectively included patients with transurethrally (TUR) resected, primary (single or multiple), non-muscle-invasive, high-grade, urothelial carcinoma of the urinary bladder. The follow-up period started on the day of the first postoperative follow-up cystoscopy (at 3 months after the original endoscopic surgery). Exclusion criteria were: present or previous upper urinary tract carcinoma and muscle-invasive disease.

The patients received a single immediate postoperative intravesical instillation of chemotherapy (Epirubicin 50 $\mathrm{mg}$ ), if there was no contraindication. Random biopsies of the bladder were performed, if there was a suspicion of carcinoma in situ (Tis). Whenever necessary (cases of incomplete resection or biopsy material without muscle fibers), a TUR was repeated within 6 weeks. All patients received an induction (6 weekly intravesical BCG instillations) as well as a 3-year maintenance BCG therapy (6). The follow-up was based on the Guidelines for "Non-muscle-invasive bladder cancer" by the European

No conflict of interest declared. 


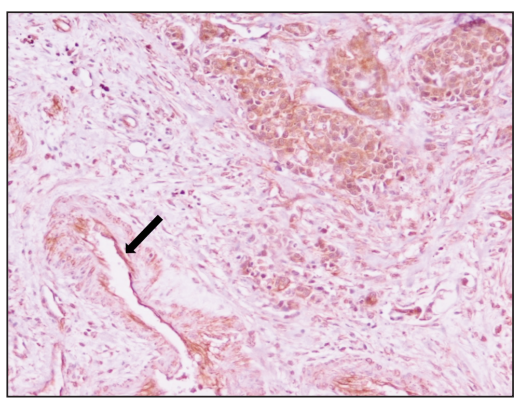

Figure 1.

High-grade nonmuscle invasive urothelial carcinoma stage pT1. Strong cytoplasmic immunostaining for ET-1 (x400). Positive marker the vascular endothelium (arrows).

Association of Urology/EAU (GEAU) (7). Histologically proven urothelial carcinoma after transurethral resection of any cystoscopic lesion found during the follow-up period was set as "disease recurrence". Any change from pTa to at least pTl and from pT1 to at least pT2 stage was set as "disease progression". Our further treatment decisions concerning recurrence and progression were based on the recommendations made by the GEAU (7). Group A consists of 40 consecutive patients with disease recurrence \pm progression. A patient was allocated in Group A as soon as recurrence was initially documented in the follow-up period. Apparently, there can be no progression without the event of recurrence, but it is not for sure that every patient with recurrence will end up in having progression. Group B includes 20 consecutive patients who had neither recurrence nor progression. Taking into account the predefined size of Group A, this size of Group B is the minimum numerical value allowing a reliable statistical analysis within a prospective framework. All included patients provided their consent. The pathology staging was done according to the 2009 TNM classification approved by the Union International Contre le Cancer (UICC) which was updated in 2017 (8 ${ }^{\text {th }}$ Edition) and grading was estimated according to the 2004 WHO grading system. A central pathology review was applied for the grading of the immunohistochemical
$=2$, strong $=3)$ in a manner consistent with previous investigations (5).

Epidemiological (age, gender, smoking), pathological (stage T, concomitant carcinoma in situ/Tis) and clinical parameters (number of tumors, tumor size, patient group), and ET-1 expression were statistically analyzed. Univariate analysis for recurrence (REC) and progression (PR) was performed using Chi-Square or Fisher's Exact test and multivariate analysis using multiple logistic regression. Univariate analysis for recurrence-free survival (RFS) and progression-free survival (PFS) was performed using Log-rank test for categorical variables and Cox regression for scale variables. Multivariate analysis for RFS and PFS was assessed using Cox regression analysis after checking the proportional hazards assumption.

The level of statistical significance was set as $\mathrm{p} \leq 0.05$.

All analyses were performed with the use of IBM SPSS Statistics version 21 software.

The study was approved by the Ethics CommitteeScientific Board of the University Hospital of Larissa and it conforms to the provisions of the Declaration of Helsinki (as revised in Tokyo 2008).

\section{RESULTS}

We totally included 60 patients, 40 patients in Group A and 20 patients in Group B. Median follow-up was 63.2 months for Group A ( $\mathrm{n}=40)$ and 87.8 months for Group $B(n=20)$. Baseline characteristics for the total number of patients as well as for the patients of Groups A and B are shown in Table 1. The results of the IHC for both Groups are summarized in Table 2 and the detailed results of the IHC regarding REC and PR for Group A are presented in Table 3.

In Group A, 27 patients had recurrence (27/40, 67.5\%) and the median RFS was 11.3 months. Moreover, 13 patients had recurrence and progression (13/40, 32.5\%) and the median PFS was 38.9 months. The patients of

\section{Table 1.}

Baseline characteristics of patients in Group A and B.

\begin{tabular}{|c|c|c|c|c|}
\hline Patients' baseline characteristics & TOTAL $(n=60)$ & GROUPA $(n=40)$ & GROUP B $(n=20)$ & $p$ value \\
\hline Median age (years) & $67.3 \pm 8.4(38-87)$ & $68.7 \pm 7.4(58-87)$ & $64.3 \pm 9.1(38-77)$ & $0.749\left(^{*}\right)$ \\
\hline$\overline{\operatorname{Gender}\left(0^{\top} / \text { P) }\right.}$ & $50 / 10(83.3 \% / 16.7 \%)$ & $34 / 6(85 \% / 15 \%)$ & $16 / 4(80 \% / 20 \%)$ & $0.356\left(^{\wedge}\right)$ \\
\hline Smoker (Yes/No/Ex) & $30 / 9 / 21(50 \% / 15 \% / 35 \%)$ & $20 / 5 / 15(50 \% / 12.5 \% / 37.5 \%)$ & $10 / 4 / 6(50 \% / 20 \% / 30 \%)$ & $0.112\left({ }^{\wedge}\right)$ \\
\hline Number of tumors (Single/Multiple) & $31 / 29(51.7 \% / 48.3 \%)$ & $20 / 20(50 \% / 50 \%)$ & $11 / 9(55 \% / 45 \%)$ & $0.471\left(^{\wedge}\right)$ \\
\hline Tumor size $(>3 \mathrm{~cm} /<3 \mathrm{~cm}$ ) & $30 / 30(50 \% / 50 \%)$ & $21 / 19(52.5 \% / 47.5 \%)$ & $9 / 11(45 \% / 55 \%)$ & $0.355\left(^{\wedge}\right)$ \\
\hline Concomitant carcinoma in situ (Yes/No) & $12 / 48(20 \% / 80 \%)$ & $8 / 32(20 \% / 80 \%)$ & $4 / 16(20 \% / 80 \%)$ & $0.642(\Lambda)$ \\
\hline Tumor stage T (Ta/T1) & $15 / 45(25 \% / 75 \%)$ & $11 / 29(27.5 \% / 72.5 \%)$ & $4 / 16(20 \% / 80 \%)$ & $0.490(\wedge)$ \\
\hline
\end{tabular}

staining (IHC). Representative samples of good morphology and antigenicity of the primary tumors were obtained for staining. Staining was performed in a single run and by applying mouse monoclonal antibody for ET-1 (clone TR.ET.48.5, dilution 1:250, NOVUS Biologicals, Littleton, Colorado, USA). Staining intensity (SI) of ET-1 on a highpower field (Figure 1) was classified according to an arbitrary four-tiered scale (no staining $=0$, mild $=1$, moderate
Table 2.

Summarized results of the immunohistochemical staining (IHCS) for Groups A and B.

\begin{tabular}{|c|c|c|c|c|c|}
\hline \multirow[t]{2}{*}{ IHCS } & \multicolumn{4}{|c|}{ Grading of IHCS } & \multirow[t]{2}{*}{ Patients' group } \\
\hline & 0 & 1 (mild) & 2 (moderate) & 3 (strong) & \\
\hline$\overline{E T-1}$ & $1(2.5 \%)$ & $18(45 \%)$ & $15(37.5 \%)$ & $6(15 \%)$ & $A(n=40)$ \\
\hline E-1 & $5(25 \%)$ & $12(60 \%)$ & $2(10 \%)$ & $1(5 \%)$ & $B(n=20)$ \\
\hline
\end{tabular}


Table 3.

Detailed results of the immunohistochemical (IHC) staining for Group A regarding recurrence and progression.

\begin{tabular}{|c|c|c|c|c|c|c|c|c|c|}
\hline \multirow[t]{2}{*}{ IHC } & \multicolumn{4}{|c|}{ Grading of IHC } & \multirow[t]{2}{*}{ Group A } & \multicolumn{4}{|c|}{$p$ value } \\
\hline & 0 & 1 (mild) & 2 (moderate) & 3 (strong) & & REC & RFS & PR & PFS \\
\hline$\overline{\mathrm{E}-1}$ & $1 / 1(100 \%)$ & $6 / 18(33.3 \%)$ & $14 / 15(93.3 \%)$ & $6 / 6(100 \%)$ & Recurrence $n=27 / 40(67.5 \%)$ & $0.000^{*}$ & $0.000^{\wedge}$ & & \\
\hline$\overline{\mathrm{E}}-1$ & $0 / 1(0 \%)$ & $2 / 18(11.1 \%)$ & $7 / 15(46.7 \%)$ & $4 / 6(66.7 \%)$ & Recurrence \& Progression $n=13 / 40(32.5 \%)$ & & & $0.000^{*}$ & $0.000^{\wedge}$ \\
\hline
\end{tabular}

Figure 2.

The expression of ET-1 in primary high-grade non-muscle-invasive urothelial bladder cancer (HGNMIUBC) significantly associates with recurrence-free survival/RFS (IHC: immunohistochemical staining).

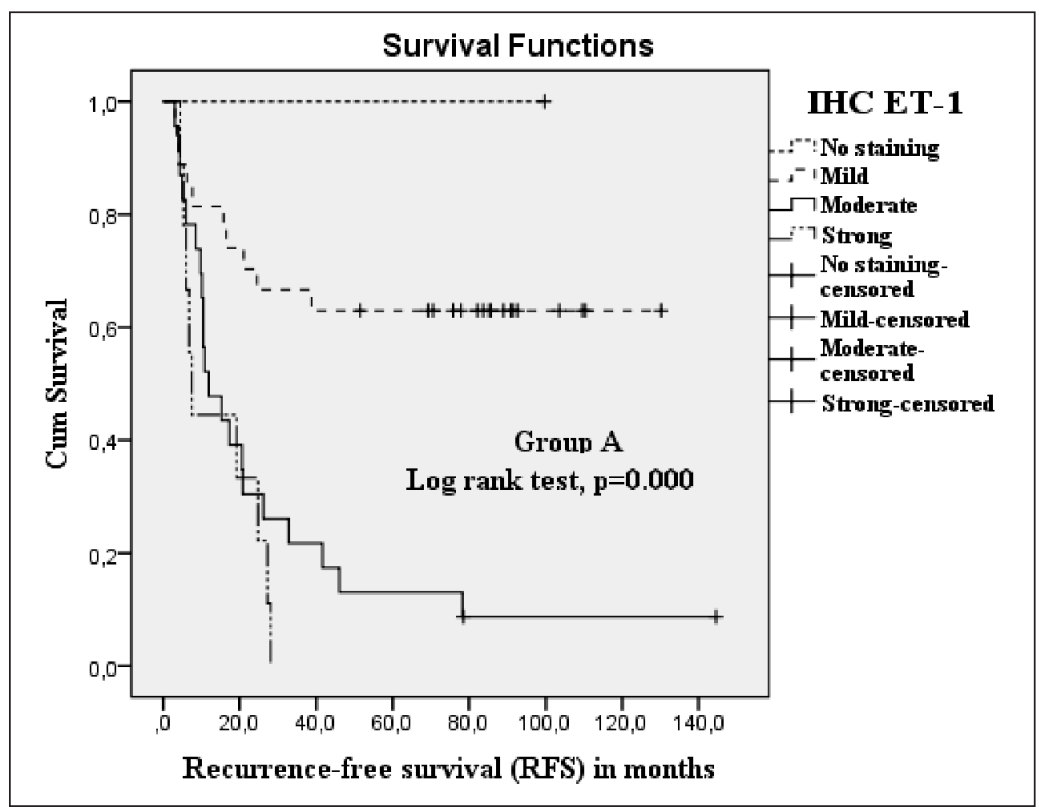

Figure 3.

The expression of ET-1 in primary high-grade non-muscle-invasive urothelial bladder cancer (HGNMIUBC) significantly associates with progression-free survival/PFS (IHC: immunohistochemical staining).

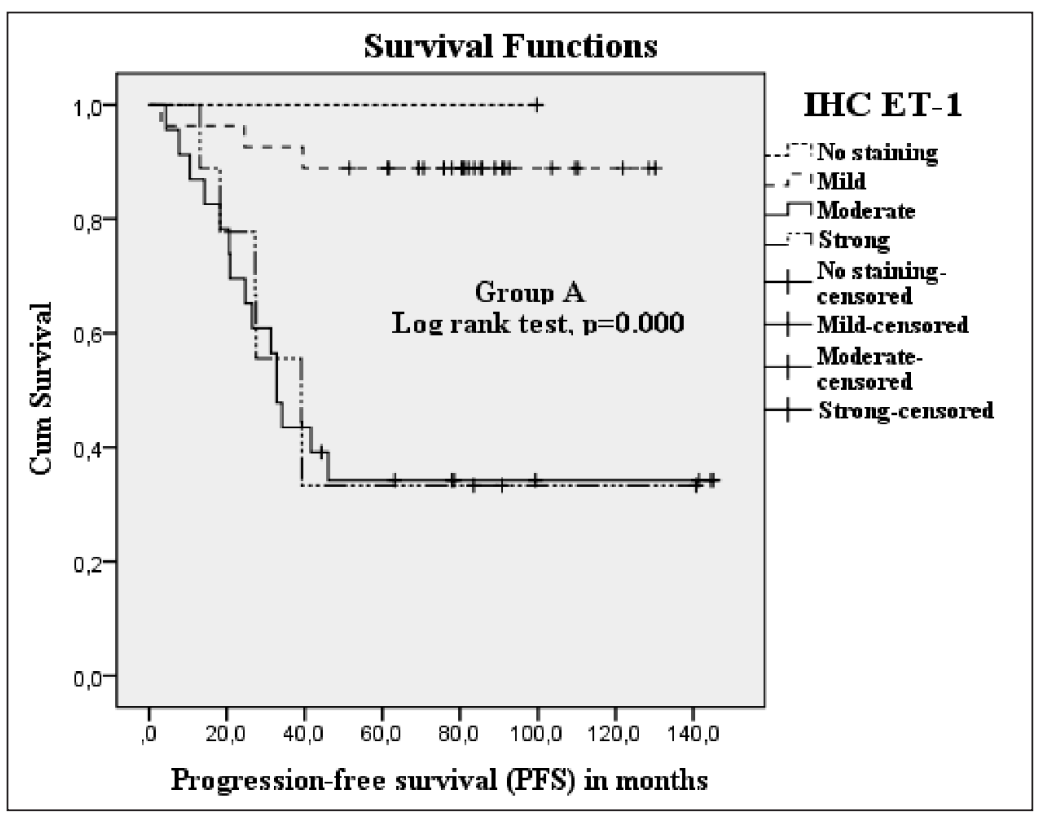

Group B had neither recurrence nor progression. Median RFS and median PFS were 87.8 months.

Recurrence: In univariate analysis, ET-1 $(\mathrm{p}=0.000)$ and age $(\mathrm{p}=0.001$, T-test $)$ were statistically significant.

Specifically, among the patients of Group A who had recurrence $(n=27 / 40), 26$ of them $(26 / 27,96.3 \%)$ showed ET-1 expression in the IHC. The multivariate analysis showed significance only for the patient's age $(\mathrm{p}=0.003, \mathrm{OR}=1.273,95 \% \mathrm{CI}$ : 1.086-1.492).

Progression: In univariate analysis, ET-1 $(\mathrm{p}=0.000)$ was significant. Notably, among the patients of Group A who had progression ( $\mathrm{n}=13 / 40), 13$ of them $(13 / 13,100 \%)$ showed ET-1 expression in the IHC. No parameter showed significance in multivariate analysis.

Recurrence-free survival (RFS): In univariate model, ET-1 was importantly associated with RFS ( $p=0.000)$, which is illustrated in the Kaplan-Meier curves (Figure 2). Age is significant too ( $\mathrm{p}=0.013$, Cox regression). The multivariate model provided no significant finding.

Progression-free survival (PFS): As it is shown by Kaplan-Meier curves (Figure 3), the univariate analysis revealed the significance of ET-1 ( $p=0.000)$. No parameter proved to be significant in multivariate analysis.

\section{Discussion}

The two major events in the natural history of HGNMIUBC are recurrence and progression to muscle-invasiveness, despite the initial high response-rate to postoperative intravesical BCG treatment (8). From a clinical point of view it is difficult and of value to identify on time and correctly those patients who will experience a failure of BCG-therapy and consequently recurrence and/or progression. Of course, the heterogeneity of the tumor does not facilitate a safe prognosis.

Risk tables for predicting recurrence and progression, based on the six most significant clinical and pathological factors, were developed by the European Organization for 
Research and Treatment of Cancer (EORTC) and represent a means whose prognostic value was confirmed by data from other studies $(7,9)$. The EORTC scoring system has not achieved a universal and unanimous acceptance though. One of the main reasons for this fact is that none of the included patients received maintenance therapy with BCG, which is currently recommended for all the high-risk patients (3). Another prognostic model was proposed from the Club Urológico Español de Tratamiento Oncológico (CUETO). In this study, patients were stratified according to the risk of recurrence and progression, which also stratified a patient's risk of recurrence after BCG plus interferon- $\alpha$ therapy (3). The maintenance scheme lasted for 5-6 months, which is considerably shorter than the actually recommended one by the EAU (3). Except for these two widespread prognostic tools, there are numerous studies, which propose different prognostic factors, including molecular ones, for categorizing patients in terms of clinical course and outcome (4). For example, Cambier et al. studied a total of 1812 Ta-T1 patients from EORTC studies 30962 and 30911 (3). Patients were allocated either to 3 years of maintenance BCG or 1 year of maintenance. The prior recurrence rate and the number of tumors were identified by multivariable analyses as statistically significant prognostic factors for recurrence (3). Tumor stage and grade were found to be important for progression and death caused by bladder cancer (3). This study had also limitations (no patients with Tis were included, routine repeat TUR resection was not performed in high-risk patients, there was no central pathology review, upper urinary tract was not investigated upon recurrence or progression, no use of biomarker). Furthermore, Palou et al. documented that female gender and carcinoma in situ in the prostatic urethra are negative prognostic factors for recurrence $(p=0.0003$, HR:2.53), progression $(p=0.001, H R: 359)$ and disease specific mortality $(\mathrm{p}=0.004$, HR: 3.53$)$ in pT1G3 bladder cancer patients treated with BCG (10). Another interesting study from Gontero et al., showed that pTlG3 patients $\geq 70$ years old with tumors $\geq 3 \mathrm{~cm}$ and concomitant carcinoma in situ should be handled aggressively because of the high risk of progression (11). Recently, Sahan et al. found in a retrospective cohort of pTl bladder urothelial carcinoma patients treated postoperatively with intravesical BCG, for at least 1 year, that the tumor invasion to the muscularis mucosaevascular plexus significantly associates with recurrence (12). In closing, no molecule is turned to be a marker until today. The available data regarding the expression of ET axis in bladder cancer does not give a clear end result. Eltze et al. examined retrospectively the expression of the endothelin axis in 154 patients with primary bladder cancer (pTa-pT2). They ascertain that both lack of ET-1 and ETAR have a negative prognostic impact (13). Moreover, an IHC study of 157 radical cystectomy samples demonstrated an overexpression of the entire ET axis. A survival benefit (disease-free survival, overall survival) was established only for ETBR (+) tumors (14). Two experimental studies, in which KU-19-19 bladder cancer cells were implanted in mice, showed that the administration of atrasentan, a selective ETAR antagonist, lead to a diminished tumor-growth rate with an increased necrosis in the tumor tissue, no significant cytoreduction and increased ETAR expression, implying an escape mechanism to overcome the antiproliferative effect caused by targeting ETAR $(15,16)$. In another IHC retrospective study, ET-1 and the microvessel density (MVD) seemed to be "good prognostic factors", cause the ET-1 overexpression was importantly associated with increased MVD and organ-confined disease (17).

Coming to an end, a prospective study of 2015, based on IHC, recorded that the overexpression of ET-1 was remarkably correlated with an increased hazard ratio of progression and death (5). In this case, the included patients suffered from non-metastatic muscle-invasive bladder cancer.

We found that the expression of ET-1 indicates a higher risk for disease recurrence and progression. Regarding the relative survival estimations (RFS, PFS), this higher risk remains documented. ET-1 could make us alert, in order to recognize the patients with primary HGNMIUBC who are at risk for a bad clinical outcome, taking into consideration that we can accurately identify patients who will not progress or die due to bladder cancer (pTaG1), but we cannot reliably identify the ones who do progress and die of their disease (3). Within this context, we could offer these patients a radical treatment early in the natural course of the disease, aiming to maximize the clinical benefit. Finally, only the patient's age, among all the rest studied parameters, correlates significantly with recurrence and RFS. So, the implementation of a strict follow up for the elderly patients with primary HGNMIUBC is a reasonable decision.

The prospective nature of our work is an important advantage. Another quality which worths to be mentioned is that our patients are the most homogenous group compared to the included patients of the abovementioned researches (different stages, different types of carcinoma). They all are primary HGNMIUBC patients treated postoperatively with intravesical BCG instillations (induction and 3-year maintenance). Likewise, we study ET-1 using IHC staining, which is the most common methodology in current literature. In our opinion, it is also positive the fact that we applied a central pathology review. On the other hand, it remains as a disadvantage the limited sample. Regarding this point, we need to mention that there is no other similar study addressing the same issues, meaning a prospective study of a rather homogenous cohort.

The standard of care in HGNMIUBC does not achieve an ideal clinical result. The prognosis also remains problematic. Taking into account, that a molecular marker could be helpful in this field, our study gives an evidence for the usefulness of ET-1 in HGNMIUBC as an alert marker for disease recurrence as well as progression.

\section{Conclusions}

ET-1 seems to deteriorate prognosis in patients suffering from primary high-grade non-muscle-invasive urothelial bladder cancer, who are treated with adjuvant BCG instillations. Furthermore, the patient's age associates with an increased likelihood for recurrence. Further studies are needed in order to clarify the potential role of 
ET-1 in the active molecular mechanism in bladder cancer and confirm or not our promising finding.

\section{ACKNOWLEDGEMENTS}

The corresponding author has been awarded a supporting funding from the Hellenic Urological Association (HUA).

\section{REFERENCES}

1. Sylvester RJ, Brausi MA, Kirkels WJ, et al. Long-term efficacy results of EORTC Genito-Urinary Group randomized phase 3 study 30911 comparing intravesical instillations of epirubicin, bacillus Calmette-Guérin, and bacillus Calmette-Guérin plus isoniazid in patients with intermediate- and high-risk stage Ta T1 urothelial carcinoma of the bladder. Eur Urol. 2010; 57:766-773.

2. Soloway M, Khoury S. Bladder Cancer. $2^{\text {nd }}$ Edition: ICUD-EAU 2012; 254-261.

3. Cambier S, Sylvester RJ, Collette L, et al. EORTC nomograms and risk groups for predicting recurrence, progression, and diseasespecific and overall survival in non-muscle-invasive stage Ta-T1 urothelial bladder cancer patients treated with 1-3 years of maintenance Bacillus Calmette-Guérin. Eur Urol. 2016; 69:60-9.

4. Mitrakas L, Gravas S, Papandreou C, et al. Primary high-grade non-muscle-invasive bladder cancer: high NF-B expression in tumor specimens distinguishes patients who are at risk for disease progression. Pathol Oncol Res. 2019; 25:225-231.

5. Mitrakas L, Gravas S, Karasavvidou F, et al. Endothelin-1 overexpression: a potential biomarker of unfavorable prognosis in nonmetastatic muscle-invasive bladder cancer. Tumour Biol. 2015; 36:4699-705. Erratum in: Tumour Biol. 2015; 36:3127

6. Lamm DL, Blumenstein BA, Crissman JD, et al. Maintenance bacillus Calmette-Guérin immunotherapy for recurrent TA, T1 and carcinoma in situ transitional cell carcinoma of the bladder: a randomized Southwest Oncology Group Study. J Urol. 2000; 163:1124-1129.

7. Babjuk M, Böhle A, Burger M, et al. EAU Guidelines on non-muscle-invasive urothelial carcinoma of the bladder: update 2016. Eur Urol. 2017; 71:447-461.

8. Zachos I, Tzortzis V, Mitrakas L, et al. Tumor size and T stage correlate independently with recurrence and progression in high-risk non-muscle-invasive bladder cancer patients treated with adjuvant BCG. Tumour Biol. 2014; 35:4185-4189.

9. Sylvester RJ, van der Meijden AP, Oosterlinck W, et al. Predicting recurrence and progression in individual patients with stage Ta T1 bladder cancer using EORTC risk tables: a combined analysis of 2596 patients from seven EORTC trials. Eur Urol. 2006; 49:466-5.

10. Palou J, Sylvester RJ, Faba OR, et al. Female gender and carcinoma in situ in the prostatic urethra are prognostic factors for recurrence, progression, and disease-specific mortality in T1G3 bladder cancer patients treated with bacillus Calmette-Guérin. Eur Urol. 2012; 62:118-25.

11. Gontero P, Sylvester R, Pisano F, et al. Prognostic factors and risk groups in T1G3 non-muscle-invasive bladder cancer patients initially treated with Bacillus Calmette-Guérin: results of a retrospective multicenter study of 2451 patients. Eur Urol. 2015; 67:74-82.

12. Sahan A, Gerin F, Garayev A, et al. The impact of tumor invasion to muscularis mucosaevascular plexus on patient outcome in pT1 bladder urothelial carcinoma. Arch Ital Urol Androl. 2020; 92:239-243.

13. Eltze E, Wild PJ, Wülfing C, et al. Expression of the endothelin axis in noninvasive and superficially invasive bladder cancer. Relation to clinicopathologic and molecular prognostic parameters. Eur Urol. 2009; 56:837-847.

14. Wülfing C, Eltze E, Yamini J, et al. Expression of the endothelin axis in bladder cancer: Relationship to clinicopathologic parameters and long-term survival. Eur Urol. 2005; 47:593-600.

15. Wülfing $C$, Tiemann A, Persigehl T, et al. In vivo activity of ABT627, a selective endothelin-A-receptor antagonist, in bladder cancer xenograft tumors. Proc Am Soc Cancer Res. 2005; 46:abstract 3039.

16. Herrmann E, Tiemann A, Eltze E, et al. Endothelin-A-receptor antagonism with atrasentan exhibits limited activity on the KU-1919 bladder cancer cell line in a mouse model. J Cancer Res Clin Oncol. 2009; 135:1455-1462.

17. Herrmann E, Bögemann M, Bierer S, et al. The role of the endothelin axis and microvessel density in bladder cancer - correlation with tumor angiogenesis and clinical prognosis. Oncol Rep. 2007; 18:133-138.

\section{Correspondence}

Lampros Mitrakas, MD, PhD (Corresponding Author)

lamprosmit@gmail.com

Roosevelt 61, 41222 Larissa (Greece)

Stavros Gravas, MD, PhD

sgravas2002@yahoo.com

Ioannis Zachos, MD, PhD

johnbzac@yahoo.gr

Anastasios Karatzas, MD, PhD

adkaratzas@yahoo.gr

Athanasios Oeconomou, MD

thaoik@hotmail.com

Vasilios Tzortzis, MD, PhD

tzorvas@otenet.gr

Department of Urology, University Hospital of Larissa,

41110 Larissa (Greece)

Foteini Karasavvidou, $\mathrm{MD}, \mathrm{PhD}$

fotkarasa@yahoo.gr

Georgios Koukoulis, MD, PhD

kougeo@med.uth.gr

Department of Pathology and Cytology, University Hospital of Larissa,

41110 Larissa (Greece)

Christos Papandreou, MD, PhD

cpapandreou@auth.gr

Department of Oncology, N.Papageorgiou Hospital of Thessaloniki

Ring Road of Thessaloniki, 56403 Thessaloniki (Greece) 